\title{
$\begin{array}{ll}\text { Research Square } & \text { Preprints are preliminary reports that have not undergone peer review. } \\ \text { They should not be considered conclusive, used to inform clinical practice, }\end{array}$ or referenced by the media as validated information. \\ Vortices Nucleation By Inherent Fluctuations In Nematic Liquid Crystal Cells
}

\section{Esteban Aguilera}

Facultad de Ciencias Físicas y Matemáticas, Universidad de Chile

Marcel G. Clerc

Facultad de Ciencias Físicas y Matemáticas, Universidad de Chile

Valeska Zambra ( $\nabla$ valeska.zambra@ug.uchile.cl)

Facultad de Ciencias Físicas y Matemáticas, Universidad de Chile https://orcid.org/0000-0002-88065719

\section{Research Article}

Keywords: Vortex solutions, nucleation process, phase singularity, noise

Posted Date: November 30th, 2021

DOl: https://doi.org/10.21203/rs.3.rs-859280/v1

License: (c) (i) This work is licensed under a Creative Commons Attribution 4.0 International License. Read Full License

Version of Record: A version of this preprint was published at Nonlinear Dynamics on April 20th, 2022. See the published version at https://doi.org/10.1007/s11071-022-07396-5. 


\title{
Vortices nucleation by inherent fluctuations in nematic liquid crystal cells
}

\author{
Esteban Aguilera - Marcel G. Clerc - Valeska Zambra
}

Received: date / Accepted: date

\begin{abstract}
Multistable systems are characterized by exhibiting domain coexistence, where each domain accounts for the different states. In the case of these systems are described by vectorial fields, domains are connected through topological defects. Vortices are one of the most frequent and studied topological defect points. Optical vortices are equally relevant for their fundamental features as beams with topological features and their applications in image processing, telecommunications, optical tweezers, and quantum information. The interaction of light beams with matter vortices in liquid crystal cells is a natural source of optical vortices. The rhythms that govern the emergence of matter vortexes due to fluctuations are not established. Here we investigate the nucleation mechanisms of the matter vortices in liquid crystal cells and establish statistical laws that govern them. Based on a stochastic amplitude equation, the law for the number of nucleated vortices as a function of anisotropy, voltage, and noise level intensity is set. Experimental observations in a nematic liquid crystal cell with homeotropic anchoring and a negative anisotropic dielectric constant under the influence of a transversal electric field show a fair agreement with the theoretical findings.
\end{abstract}

Keywords Vortex solutions · nucleation process · phase singularity $\cdot$ noise

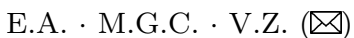

Physics Department and Millennium Institute for Research in Optics, Facultad de Ciencias Físicas y Matemáticas, Universidad de Chile, Casilla 487-3, Santiago, Chile.

E-mail: valeska.zambra@ug.uchile.cl

\section{Introduction}

Out-of-equilibrium systems often exhibit multistability, that is, by setting the parameters of the system, and for different initial conditions, these systems can exhibit different equilibrium states $[1,2]$. In the case that these equilibria account for the orientation of some physical quantities, the union of three different domains is characterized by exhibiting a vortex $[1,3]$. These vortices correspond to points or lines where the magnitude of the vector quantity is zero, and its respective phase value is undefined, phase singularity. Examples of everyday vortices are eddies or tornadoes in fluids, hair whorl, umbilic defects in liquid crystals, and skyrmions in magnetic systems.

In the last decades, a great effort has been developed to understand spiral out light beams about their axis of propagation, orbital angular momentum of light or optical vortex [4-8]. These beams have a donut-like structure, that is, the beam intensity cancels out into the center, generating a phase singularity into the envelope. In addition, the light beams are characterized by fading asymptotically from the center. Around the point of zero intensity, the phase distribution forms an $N$-armed spiral, with $N$ being the topological charge [3, $5-8]$. These optical vortices have aroused interest from both the fundamental and applied point of view. The photonic applications ranging from optical tweezers [911], enhancement of astronomical images [12], quantum computation [13], wavefront sensors [14], and data transmission [15]. From a fundamental point of view, the interchange of angular momentum between light and matter has attracted attention (see the collected articles [8] and references therein). Different methods have been used to generate optical vortices based on diffractive elements [16], deformable mirrors [17], holo- 


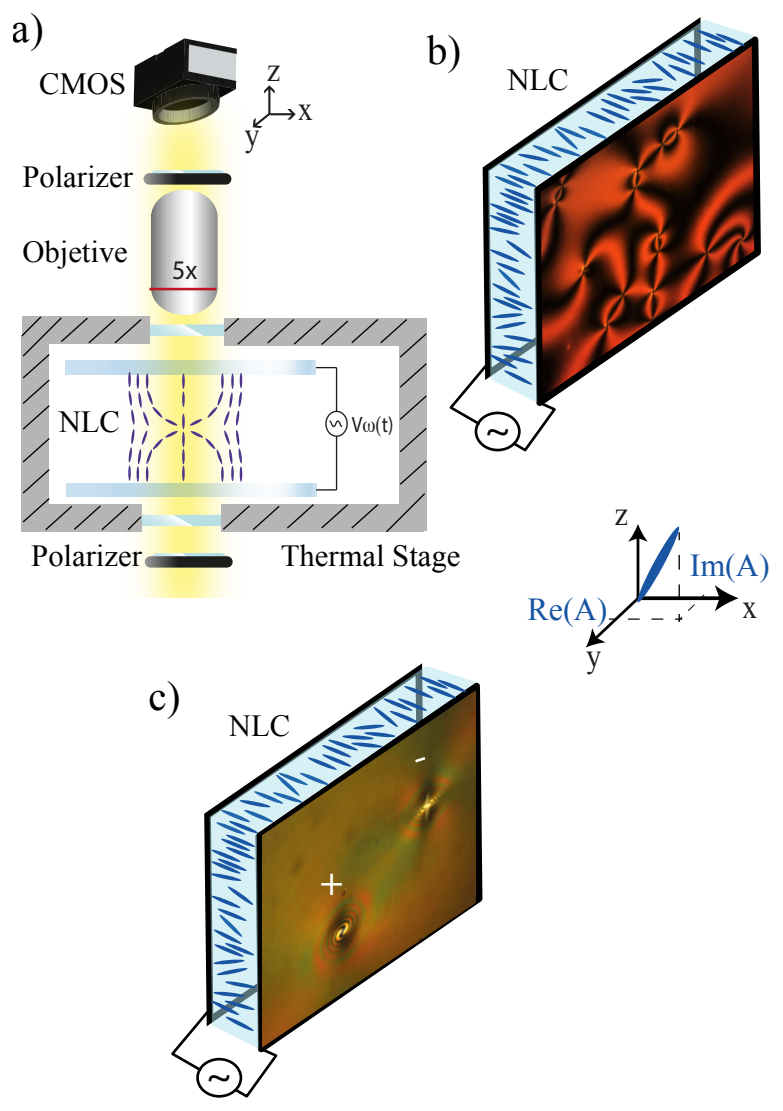

Fig. 1 Vortex nucleation in a nematic liquid crystal light cell with homeotropic anchoring and negative dielectric anisotropic constant. a) Schematic representation of the experimental setup. b) Snapshot of a vortex gas obtained in the liquid crystal cell. The lower inset is a schematic representation of the director and its associated complex amplitude. c) Vortices of positive and negative charge observed with crossed circular polarizers.

grams [18], spiral phase plates [19], nanostructured glass plates [20], and helical structures of liquid crystals [2124]. In most of these methods, the light beam interacts with a material object which has an intrinsic helicity. Hence, to control the optical vortex, it is important to have an adequate alignment between the light beam, the helical target, and the geometry of it. In the case of liquid crystals with photosensitive walls, the light induces a vortex in the matter (umbilical defect), with which interacts, generating an optical vortex [24, 25]. The vortex-like defects have accompanied liquid crystals since their discovery in 1889 by Lehmann [26]; later, Friedel solved its detailed topological structure [27], which was complemented by the elastic theory of crystals by Frank [28]. Because of the topological structure of these defects corresponding to a rod-like object in three dimensions, these defects are usually called nematic umbilical defects [29]. The dynamics and properties of umbilic defects can be described employing the
Frank-Oseen free energy [30,31]. However, a more accessible approach that captures the dynamics of umbilic defects is through the Ginzburg-Landau amplitude equation with real coefficients $[25,32]$. When a sufficiently large electric field is applied to a nematic liquid crystal cell with homeotropic anchoring and a negative anisotropic dielectric constant, a gas of umbilical defects emerges (see Fig. 1). These defects later begin to be annihilated by pairs with opposite charges to minimize the Free energy [30]. Figure 1c shows two vortices of opposite charge under circular crossed polarizers. To our knowledge, the emergence process and statistical rules governing this phase singularity gas have not been established.

The paper aims to establish the nucleation mechanisms of the matter vortices in nematic liquid crystal cells and provide statistical laws that govern them. Based on a stochastic amplitude equation, the Ginzburg Landau equation with additive noise, we establish the law for the number of nucleated vortices as a function of anisotropy, bifurcation parameter, and intensity of the noise level. Experimentally, we consider a nematic liquid crystal cell with homeotropic anchoring and a negative anisotropic dielectric constant under the influence of a transverse electric field. The average number of umbilical defects as a function of the applied voltage and temperature shows a good agreement with the theoretical findings.

The article has the following structure: liquid crystal cells, the experimental setup, and experimental vortices nucleation and annihilation is described in Sec. 2. The amplitude equation that accounts for the reorientation dynamics of a nematic liquid crystal cell is presented in Sec. 3. In addition, a numerical and experimental analysis of vortex nucleation is presented. Our conclusions and comments are left for the final section.

\section{Nematic liquid crystal cell}

Nematic liquid crystals are nonlinear optical media [30, 31 , composed by rod-like molecules that have a preferential orientation order but not a positional one. This state of matter shares features of solids and liquids, such as fluidity and birefringence. Introducing a liquid crystal inside a cell, that is, it is sandwiched between two confining glass layers, the molecules are oriented according to anchoring conditions. Homeotropic anchoring is characterized by molecules that are oriented orthogonal to cell walls, as illustrated in Fig. 1a. If the dielectric anisotropic constant of the liquid crystal is negative, when applying a vertical electric field, the molecules tend to orient orthogonal to it. The elastic and electric energy determines the equilibrium angle 

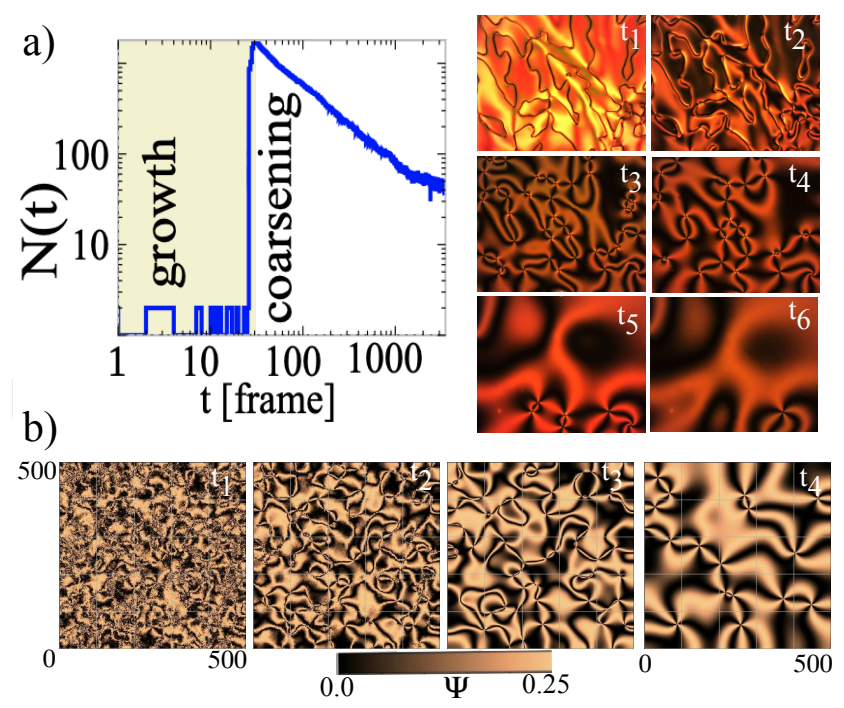

Fig. 2 Nucleation and evolution of umbilical defects in a liquid crystal cell driven by an electric field. a) Experimental temporal evolution of the number of vortices as a function of time. Right panel account for a temporal sequence of snapshots of the liquid crystal cell driven by an electric field and constant temperature $\left(t_{1}<t_{2}<t_{3}<t_{4}<\right.$ $\left.t_{5}<t_{6}\right)$. b) Numerical temporal sequence of polarized field $\Psi(\vec{r}, t)=\operatorname{Re}(A) \operatorname{Im}(A)$ obtained by the numerical simulations of the stochastic Ginzburg-Landau equation (1) with $\mu=1.0$, $\delta=0.0$, and $T=0.01\left(t_{1}<t_{2}<t_{3}<t_{4}\right)$.

with respect to the vertical axis. This generates different domains connected by orientation defects or phase singularities, matter vortices [30]. Figure $1 \mathrm{~b}$ shows the umbilical defects observed in a liquid crystal nematic cell. The defects correspond to the intersection of two black fringes.

\subsection{Experimental setup}

Let us consider a $15 \mu \mathrm{m}$ thick cell, (SB100A150uT180 manufactured by Instec), filled with nematic liquid crystal LC BYVA- 01 (Instec) with dielectric anisotropy $\epsilon_{a}=-4.89$, birefringence $\Delta n=n_{e}-n_{o}=0.1$, rotation viscosity $\gamma=204 \mathrm{mPas}$, splay and bend elastic constant, respectively, $K_{1}=17.65 \mathrm{pN}$ and $K_{3}=21.39 \mathrm{pN}$. This liquid crystal cell is placed inside a thermal control chamber (Linkam LTS420), which in turn is inserted inside a microscope (Leica DM2700P), in between the crossed linear polarizers. Figure 1a shows a schematic representation of the experimental setup. To monitor the images a CMOS camera is connected to the microscope. A sinusoidal voltage with a frequency of $100 \mathrm{~Hz}$ is applied to the sample.

\subsection{Experimental vortices nucleation}

Maintaining the temperature at $26^{\circ} \mathrm{C}$, the voltage is turned on, the dynamics of vortex nucleation and annihilation are recorded. Figure 2a depicts the temporal evolution of the observed umbilical defects. To figure out vortex evolution, we have considered a voltage sweep between 9.0 Vpp and 30.0 Vpp. Likewise, keeping the voltage at $15 \mathrm{Vpp}$ it is switched on and sweeping the temperature between $25^{\circ} \mathrm{C}$ and $80^{\circ} \mathrm{C}$, the dynamics of vortex nucleation is analyzed. From the chart in Fig. 2a, we infer that there is an abrupt process of vortex nucleation. Once the voltage is turned on, we immediately observe the appearance of domains. These domains are separated by interfaces that are easily recognized by the camera (see inset $t_{1}$ in Fig. 2a). All these different and complicated domains are a consequence of inert thermal fluctuations, which cause the molecules to orient themselves in different directions transverse to the applied electric field. Later the domain interfaces are destabilized, through the emergence of vortices (see inset $t_{2}$ in Fig. 2a). The above process occurs in fractions of a second. Hence, vortices quickly emerge, as illustrated in inset $t_{3}$. Once established a vortex gas, the vortices are subsequently annihilated by pairs of opposite charges (see insets $t_{4}, t_{5}$ and $t_{6}$ ), generating a coarsening processes characterized by a Power Law [33].

In brief, once the voltage is applied to the nematic liquid crystal cell, vortices are generated rapidly by thermal agitation and evolve through a pair interaction process.

\section{Theoretical description of the vortices nucleation}

To shed light on the vortex nucleation mechanisms, theoretically, we consider the liquid crystal cell close to reorientation instability, which is described by the dimensionless stochastic Ginzburg-Landau equation [25, $32,34]$

$\partial_{t} A=\mu A-|A|^{2} A+\nabla^{2} A+\delta \partial_{\eta, \eta} \bar{A}+\sqrt{T} \zeta(\vec{r}, t)$,

where the complex field $A(\vec{r}, t)$ accounts for the amplitude of the critical elastic mode that describes the deviation of the molecular director with respect to the vertical direction. $\bar{A}$ accounts for the complex conjugate of $A$. $\mu$ is the bifurcation parameter that is proportional to the voltage minus the critical Fréederickz voltage $[25,32,34]$. Which is related to the physical parameters as follows

$\mu=-K_{3} \frac{\pi^{2}}{d^{2}}-\epsilon_{a} \frac{V^{2}}{d^{2}}$, 
where $d$ is the cell thickness and $V$ is the applied voltage. The temporal and spatial scales are in units of rotation viscosity $\gamma$ and elastic constants $K_{1}+K_{2}\left(K_{2}\right.$ twist elastic constant), respectively. $\delta=K_{1}-K_{2} /\left(K_{1}+K_{2}\right)$ is the parameter that accounts for the elastic anisotropy of the nematic liquid crystal. $\partial_{\eta} \equiv \partial_{x}+i \partial_{y}$ is a differential operator, note that the Laplacian operator satisfies $\nabla^{2}=\partial_{\eta, \bar{\eta}} \cdot \zeta(\vec{r}, t)$ is a Gaussian white noise with zero mean value $\langle\zeta\rangle=0$ and correlation $\left\langle\zeta(\vec{r}, t) \bar{\zeta}\left(\vec{r}^{\prime}, t^{\prime}\right)\right\rangle=$ $\delta\left(t-t^{\prime}\right) \delta\left(\vec{r}-\vec{r}^{\prime}\right)$ and $T$ accounts for the noise intensity level. A detailed derivation of the model Eq. (1) and its parameters from the dynamics of the director is presented in Ref. [25].

\subsection{Theoretical vortices nucleation}

For $\mu \leqslant 0$, the Ginzburg-Landau Eq. (1) has a null solution $A=0$ as a stable equilibrium, which corresponds to molecules are not reoriented, that is, homeotropic state is the stable configuration. For $\mu>0$, the state homeotropic state $A=0$ becomes unstable by means of a degenerate pitchfork bifurcation, giving rise to the appearance of vortices [34]. This instability is a secondorder transition that originates different domains of equilibria $A=\sqrt{\mu} e^{i \theta_{0}}$ where $\theta_{0}$ is an arbitrary constant. This instability is well-known as the Fréedericksz transition [35]. In this regime of parameters, the stochastic fluctuations induce different domains connected by point defects (phase singularities). Figure $2 \mathrm{~b}$ shows the emergence of vortices in model Eq. (1) as a result of stochastic fluctuations. To compare with the experimental observations, figure $2 \mathrm{~b}$ shows a color map of the auxiliary field $\Psi \equiv \operatorname{Re}(A) \operatorname{Im}(A)$, usually called the polarization field [25]. Note that $\Psi(\vec{r}, t)$ vanishes for any of nullclines of $A, \operatorname{Re}(A)=0$ or $\operatorname{Im}(A)=0$. The intersection of two nullcline, black fringes in Fig. 2b, accounts for a vortex. As in the experiment, initially the noise nucleates a large number of vortices (see the inset $t_{1}$ in Fig. 2b), which are subsequently annihilated by opposite pairs. The dynamics of vortex annihilation followed a coarsening process [3], which is illustrated in the sequence of colormaps in Fig. 2b. It is important to note that the amplitude equation does not fully describe all the dynamics observed experimentally. In particular, model Eq. (1) does not account for the initial creation of domains. However, it gives an adequate description of the vortices and their respective dynamics. Numerical simulations of the model Eq. (1) were implemented using a finite differences scheme in space that uses a centered stencil of five grid points with Runge-Kutta order-4 algorithm, with a 500x500 points grid temporal step $d t=0.0004$, and Neumann boundary conditions.
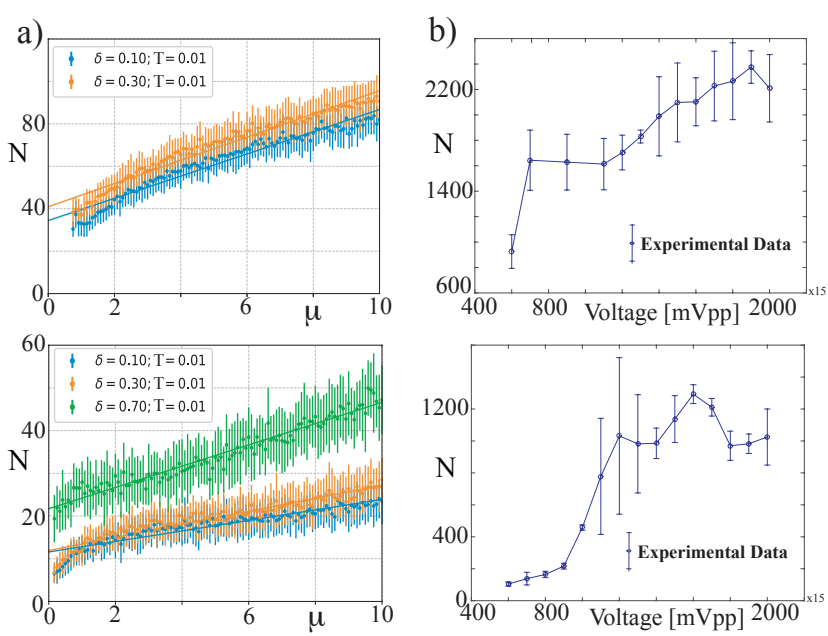

Fig. 3 The number of defects in a given instant as a function of the bifurcation parameter. a) The number of defects obtained from numerical simulations of Eq. (1) with $\mu=1.0$ at $t=12$ (top panel) and $t=60$ (bottom panel). The points with a bar account for mean value and standard deviation obtained after carrying out for each parameter 30 realizations. b) Number of umbilical defects as a function of the driven voltage at $t=0.5 \mathrm{~s}$ (top panel) and $t=1.0 \mathrm{~s}$ (bottom panel). The points with a bar account for mean value and standard deviation obtained after 5 experimental realizations.

Numerically, we have monitored the number of vortices at a given instant as a function of the bifurcation parameter $\mu$. Figure 3a summarizes the results found. From these charts, we infer that the number of vortices grows linearly with the bifurcation parameter. Likewise, we note that this behavior is not substantively modified when we change the anisotropy $\delta$. To compare and validate this numerical result, we have experimentally studied the number of umbilical defects $N$ at an instant as a function of the voltage applied to the liquid crystal sample. We found that the number of defects grows with the voltage, which shows a qualitative agreement with the numerical results (cf. Fig. 3b). The defects emerge from the homeotropic state, due to the inherent fluctuations of the system. The imperfections in the experiment, electronic noise, and elements ignored in our theory, such as black flow and movements of charges, may be responsible for the differences in fine-tuning between simulations and experiments.

\subsection{Statistical law of vortex nucleation}

To figure out the nucleation process, we approximate the model Eq. (1) by its deterministic linear part and consider the Fourier mode decomposition

$A(x, y, t)=A_{k} e^{\sigma t+i\left(k_{x} x+k_{y} y\right)}$, 
after straightforward calculations, we get

$\sigma=\mu-k_{x}^{2}(1+\delta)-k_{y}^{2}(1-\delta) \pm 2 i \delta k_{x} k_{y}$

where $R e[\sigma] \equiv \mu-k_{x}^{2}(1+\delta)-k_{y}^{2}(1-\delta)$ is the growth rate mode, $k_{x}$ and $k_{y}$ are wavenumber modes in the horizontal directions. The $\operatorname{Im}[\sigma]= \pm 2 \delta k_{x} k_{y}$ accounts for the dispersion relation. the condition $\operatorname{Re}\left[\sigma\left(k_{x}, k_{y}\right)\right]>0$ corresponds to unstable spatial modes. Notice that white noise is characterized by excited in the same manner all modes [36]. Indeed, the stochastic fluctuations of a white noise on average excite all spatial modes in the same manner. The boundary conditions and geometric dimensions of the system determine the wavenumbers of modes. For simplicity, if we consider periodic boundary conditions and a square domain wavenumbers take the form $k_{x}=2 \pi n / L$ and $k_{y}=2 \pi m / L$, where $L$ is the size of the box and $\{n, m\}$ are integer numbers. Then, the amplitude of a mode $(n, m)$ can be written in the form

$R e\left[A_{(n, m)}\right]=a_{0} \cos \left(\frac{2 \pi n}{L} x+\phi_{0}\right) \sin \left(\frac{2 \pi m}{L} y+\phi_{1}\right)$,

where $a_{0}, \phi_{0}$, and $\phi_{1}$ are constants characterizing the spatial mode. The nodes of the spatial modes correspond to zeros of the amplitude, $A_{(n, m)}=0$; that is, these nodes correspond to phase singularities for the spatial modes. The spatial mode with the maximum number of vortices (nodes) corresponds to $\operatorname{Re}(\sigma)=0$. To calculate this maximum number of vortices, we proceed by calculating the number of nodes in one direction

$\operatorname{Re}\left[\sigma\left(n^{c}, k_{y}=0\right)\right]=\mu-\left(\frac{2 \pi n^{c}}{L}\right)^{2}(1+\delta)=0$.

Then the critical number of nodes is

$n^{c}=\left(\frac{L}{2 \pi}\right) \sqrt{\frac{\mu}{(1+\delta)}}$.

Applying the same condition in the other direction, that is, $\operatorname{Re}\left[\sigma\left(k_{x}=0, m^{c}\right)\right]=0$, we get

$m^{c}=\left(\frac{L}{2 \pi}\right) \sqrt{\frac{\mu}{(1-\delta)}}$.

Finally, we determine the maximum number of vortices (nodes) of the critical spatial modes by

$N=n^{c} m^{c}=\left(\frac{L}{2 \pi}\right)^{2} \frac{\mu}{\sqrt{\left(1-\delta^{2}\right)}}$.

Notice that all other unstable modes also have a number of nodes proportional to the previous expression multiplied by a proper fraction. As we have mentioned, the stochastic fluctuations generated by white
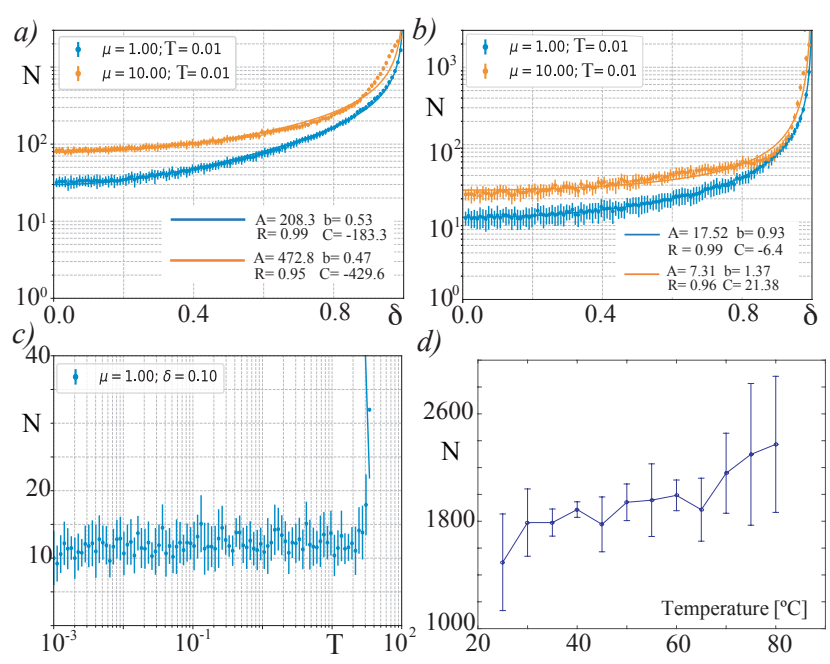

d)

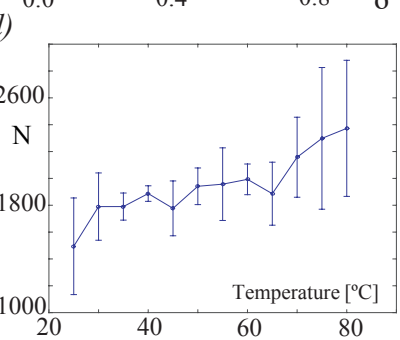

Fig. 4 The number of defects in a given instant as a function of the anisotropic parameter $\delta$. obtained from numerical simulations of Eq. (1) at $t=12$ (a) and $t=60$ (b). The points with a bar account for mean value and standard deviation obtained after carrying out for each parameter 20 realizations. The continuous curves were obtained using the fitting function $N=A /\left(1-\delta^{2}\right)^{b}+C$. The simulations and fitting parameters are specified in insets. c) The number of defects in a given moment as a function of the noise intensity level $T$. d) Umbilical defects number as a function of the temperature after 1 second of applying voltage $15 \mathrm{Vpp}$. The points with a bar account for mean value and standard deviation obtained after carrying out 5 experimental realizations.

noise excite both the stable and unstable modes in the same manner. On the other hand, the unstable modes are damped, and the stable ones grow as a consequence of the linear dynamics of the model Eq. (1). Hence, the number of vortices is proportional to the previous expression, in particular to the bifurcation parameter. Which is consistent with what is observed numerically and experimentally (see Fig. 3). Likewise, we note that expression (9) predicts that the number of vortices diverges when $\delta$ tends to 1 . This result is natural from a physical point of view, because if $\delta^{2}=1$, then some of the elastic constants diverge or disappear, which corresponds to a transition from a nematic liquid crystal to another matter state [30]. Figure 4 shows the number of vortices at a given moment as a function of the anisotropy parameter $\delta$. This type of result shows an excellent agreement with expression (9). To study its trend, we have used a more general fitting function of the form $N=A /\left(1-\delta^{2}\right)^{b}+C$, which can take into account the nonlinear effects and errors of the vortex measurement method. From charts, $4 \mathrm{a}$ and $4 \mathrm{~b}$, note that the critical exponent $b$ evaluated at higher times is dissimilar that predicted theoretically. This effect is due to the fact that nonlinear terms begin to play a non-negligible role. Note that formula (9) only contains the effects of linear theory. Experimentally, we cannot carry out a 
similar analysis since elastic anisotropy $\delta$ is determined by intermolecular interactions that we cannot control.

Formula (9) does not depend on the noise intensity level $T$. Indeed, the number of vortices (nodes) does not depend on the intensity of the noise, however, their presence is essential to stimulate unstable modes. Figure $4 \mathrm{c}$ shows that effectively the noise intensity level does not affect the number of vortices created. When the noise intensity is very large, $T>20$, the deterministic linear theory is no longer valid and the vortices are no longer related to the linear modes (see Fig. 4c). Indeed, for these level of noise values the system can be approximated by a purely stochastic one. Experimentally to study the effect of the inherent fluctuations of our physical system, we have studied the number of vortices in a given moment as a function of temperature. Figure $4 \mathrm{~d}$ summarizes the results found. We infer that there is a tendency to increase the number of vortices with temperature. The increase in temperature has a double effect; on the one hand, it increases the thermal fluctuations and, in turn, modifies the elastic constants [37]. This combine effect is responsible for the increase found in the number of vortices.

\section{Conclusions and remarks}

During the last decades, much effort has been focused on understanding topological defects and their dynamics. However, the emergency processes of these intriguing solutions have been scarcely addressed. Based on linear theory and stochastic fluctuations, we can establish that the matter vortices are a consequence of the different excited unstable spatial modes. The above is summarized by formula (9) multiplied by a constant that accounts for the effect of all unstable modes. Therefore, we can establish that the number of vortices grows proportional to the bifurcation parameter; it is inverse to the square of the elastic anisotropy and does not depend on the level of the noise intensity. Experimental observations show a good qualitative agreement with theoretical findings.

The number of vortices for long times, where the nonlinear theory governs the dynamics of the system, can no longer be given by formula (9) since the interaction of the vortex pair begins to annihilate vortices, as illustrated in Fig. 2. The complete expression of the number of vortices as a function of time is an open problem.

\section{Acknowledgments}

The authors thank Enrique Calisto, Michal Kowalczyk, and Michel Ferre for fructified discussions. This work was funded by ANID-Millennium Science Initia- tive Program-ICN17_012. MGC is thankful for financial support from the Fondecyt 1210353 project.

Data availability The datasets generated during the current study are available from the corresponding author on reasonable request.

\section{Declarations}

Conflict of interest The authors declare that they have no conflict of interest.

\section{References}

1. Pismen, L.M.: Patterns and Interfaces in Dissipative Dynamics. Springer, Berlin (2006)

2. Cross, M., Greenside, H.: Pattern Formation and Dynamics in Nonequilibrium Systems. Cambridge University Press, New York (2009)

3. Pismen, L. M.: Vortices in Nonlinear Fields. Oxford Science, (1999)

4. Sommerfeld, A.: Lectures on theoretical physics: Optics, Vol. IV, Academic press, New York, (1954)

5. Nye, J., Berry, M.: Dislocations in wave trains. Proc. R. Soc. Lond. A. 336, 165-190 (1974)

6. Allen, L., Beijersbergen, M. W., Spreeuw, R. J. C., Woerdman J.P., Orbital angular momentum of light and the transformation of Laguerre-Gaussian laser modes. Phys. Rev. A 45, 8185-8189 (1992)

7. Soskin, M.S., Vasnetov, M.V.: Progress in Optics, E. Wolf, ed. Elsevier, Vol. 42, 219-276 (2001)

8. Allen, L., Barnett, S.M., Padgett, M. J.: Optical angular momentum. CRC press, Boca Raton (2003)

9. Grier, D. G.: A revolution in optical manipulation. Nature 424, 810 (2003).

10. Shvedov, V. G., Rode, A.V., Izdebskaya, Y.V., Desyatnikov, A. S., Krolikowski, W., Kivshar, Y. S.: Giant optical manipulation. Phys. Rev. Lett. 105, 118103 (2010).

11. Padgett, M., Bowman, R.: Tweezers with a twist. Nat. Photonics 5, 343 (2011).

12. Tamburini, F., Anzolin, G., Umbriaco, G., Bianchini, A., Barbieri, C.: Overcoming the Rayleigh criterion limit with optical vortices. Phys. Rev. Lett. 97, 163903 (2006).

13. Arnaut, H.H., Barbosa, G.A.: Orbital and intrinsic angular momentum of single photons and entangled pairs of photons generated by parametric down-conversion. Phys. Rev. Lett. 85, 286 (2000).

14. Murphy, K., Dainty, C.: Comparison of optical vortex detection methods for use with a Shack-Hartmann wavefront sensor. Opt. Express 20, 4988 (2012).

15. Wang, J., Yang, J.-Y., Fazal, I.M., Ahmed, N., Yan, Y., Huang, H., Ren, Y., Yue, Y., Dolinar, S., Tur, M., Willner, A.E.: Terabit free-space data transmission employing orbital angular momentum multiplexing Nat. Photonics 6, 488 (2012).

16. Bazhenov, V.Y., Vasnetsov, M. V., Soskin, M. S.: Laser beams with screw dislocations in their wavefronts. JETP Lett. 52, 429-431 (1990).

17. Tyson, R. K., Scipioni, M., Viegas, J.: Generation of an optical vortex with a segmented deformable mirror. Appl. Opt. 47, 6300-6306 (2008).

18. Arlt, J., Dholakia, K., Allen, L., Padgett, M. J.: The production of multiringed Laguerre-Gaussian modes by computer-generated holograms. J. Mod. Opt. 45, 12311237 (1998). 
19. Beijersbergen, M. W., Allen,L., van der Veen, H.E.L.O., Woerdman, J. P.: Astigmatic laser mode converters and transfer of orbital angular momentum. Opt. Commun. 96, 123-132 (1993).

20. Beresna, M., Gecevicius, M., Kazansky, P. G., Gertus, T.: Radially polarized optical vortex converter created by femtosecond laser nanostructuring of glass. Appl. Phys. Lett. 98, 201101 (2011).

21. Voloschenko, D., Lavrentovich, O.D.: Optical vortices generated by dislocations in a cholesteric liquid crystal. Opt. Lett. 25, 317319 (2000).

22. Marrucci, L., Manzo, C., Paparo, D.: Optical spin-toorbital angular momentum conversion in inhomogeneous anisotropic media. Phys. Rev. Lett.96, 163905 (2006).

23. Brasselet, E., Murazawa, N., Misawa, H., Juodkazis, S.: Optical vortices from liquid crystal droplets. Phys. Rev. Lett. 103, 103903 (2009).

24. Barboza, R., Bortolozzo, U., Assanto, G., VidalHenriquez, E., Clerc, M.G., Residori, S.: Vortex induction via anisotropy stabilized light-matter interaction. Phys. Rev. Lett. 109, 143901 (2012).

25. Barboza, R., Bortolozzo, U., Clerc, M.G., Residori, S., Vidal-Henriquez, E.: Optical vortex induction via lightmatter interaction in liquid-crystal media Adv. Opt. Photonics 7, 635-683 (2015).

26. Lehmann O.: Über fliessende krystalle. Z. Phys. Chem. 4, 462-472 (1889).

27. Friedel G.: Les états mésomorphes de la matiere. Annales de Physique 18, 273-474 (1922).

28. Frank F. C.: Liquid crystals. On the theory of liquid crystals. Disc. Faraday Soc. 25, 19-28 (1958).

29. Rapini A. J.: Umbilics: static properties and shearinduced displacements. Physique 34, 629-633 (1973).

30. Chandrasekhar S.: Liquid Crystals. Cambridge University, New York (1977).

31. Blinov LM.: Structure and Properties of Liquid Crystals. Springer, New York, (2011).

32. Frisch, T., Rica, S., Coullet, P., Gilli, J. M.: Spiral waves in liquid crystal. Phys. Rev. Lett. 72, 1471-1474 (1994).

33. Zambra, V., Clerc, M.G., Barboza, R., Bortolozzo, U., Residori, S.: Umbilical defect dynamics in an inhomogeneous nematic liquid crystal layer. Phys. Rev. E. 101, 062704 (2020).

34. Clerc, M.G., Vidal-Henriquez, E., Davila J.D., Kowalczyk, M.: Symmetry breaking of nematic umbilical defects through an amplitude equation. Phys. Rev. E 90, 012507 (2014).

35. Fréedericksz, V., Zolina, V.: Forces causing the orientation of an anisotropic liquid. Trans. Faraday Soc. 29, 919930 (1933).

36. García-Ojalvo, J., Sancho, J.: Noise in spatially extended systems. Springer Science \& Business Media, New York, (2012).

37. Chevallard, C., Clerc, M.C.: Inhomogeneous Fréedericksz transition in nematic liquid crystals. Phys. Rev. E, 65, 011708 (2001). 